\title{
Robust Solution Procedure for the Discrete Energy-Averaged Model on the Calculation of 3D Hysteretic Magnetization and Magnetostriction of Iron-Gallium Alloys
}

\author{
Hafez Tari $^{1}$, J. J. Scheidler ${ }^{2}$, M. J. Dapino ${ }^{3}$ \\ Department of Mechanical and Aerospace Engineering, The Ohio State University, Columbus, Ohio 43210, USA
}

\begin{abstract}
A reformulation of the Discrete Energy-Averaged model for the calculation of 3D hysteretic magnetization and magnetostriction of iron-gallium (Galfenol) alloys is presented in this paper. An analytical solution procedure based on an eigenvalue decomposition is developed. This procedure avoids the singularities present in the existing approximate solution by offering multiple local minimum energy directions for each easy crystallographic direction. This improved robustness is crucial for use in black-box finite element codes. Analytical simplifications of the 3D model to 2D and 1D applications are also presented. In particular, the 1D model requires calculation for only one easy direction, while all six easy directions must be considered for general applications. Compared to the approximate solution procedure, it is shown that the resulting robustness comes at no expense for $1 \mathrm{D}$ applications, but requires almost twice the computational cost for $3 \mathrm{D}$ applications. To find model parameters, we employ the average of the hysteretic data, rather than anhysteretic curves, which would require additional measurements. An efficient optimization routine is developed that retains the dimensionality of the prior art. The routine decouples the parameters into exclusive sets, some of which are found directly through a fast preprocessing step to improve accuracy and computational efficiency. The effectiveness of the model is verified by comparison with existing measurement data.
\end{abstract}

Key words:

Iron-Gallium Alloys, Galfenol, Constitutive Modeling, Discrete Energy-Averaged model, Magnetostriction, Hysteresis.

\footnotetext{
${ }^{1}$ E-mail: tari.1@osu.edu.

${ }^{2}$ E-mail: scheidler.8@osu.edu.

${ }^{3}$ Author to whom correspondence should be addressed. E-mail: dapino.1@osu.edu.
} 


\section{Introduction}

Magnetostrictive materials undergo dimensional changes when exposed to a magnetic field, and exhibit magnetization changes when subjected to external stress. Design and optimization of magnetostrictive systems using finite element techniques require constitutive models that are robust and valid for arbitrary magnetic field and stress inputs. The robustness of each constitutive model is dictated by the complexity of the material behavior.

Galfenol (FeGa) is a common magnetostrictive material that was invented at the Naval Surface Warfare Center [1]. Galfenol is well suited for actuation and sensing applications as it possesses high tensile strength $(\sim 500 \mathrm{MPa})$, demonstrates moderate magnetostriction ( $2350 \mathrm{ppm})$ under very low magnetic fields $(\sim 8 \mathrm{kA} / \mathrm{m})$, and exhibits limited temperature dependence $([2])$ in its active properties between -20 and $80{ }^{\circ} \mathrm{C}$. In addition, Galfenol has very low hysteresis, a high Curie temperature $\left(675^{\circ} \mathrm{C}\right)$, and corrosion resistance in aqueous environments $[2,3]$. Galfenol further benefits from its moderate machinability and ductility, and its relatively inexpensive constituent materials. A detailed review of these alloys is presented by Atulasimha and Flatau [2].

Due to the diversity of active effects and the nonlinearities that magnetostrictive materials exhibit, modeling their behavior for arbitrary stress and magnetic field inputs is challenging. A wide range of models have been proposed. At one extreme, a phenomenological approach fits a curve or surface to the measurement data, which provides efficiency but ignores the underlying physics. At the other extreme, micromagnetic models consider all known energies and are very accurate. Macroscopic models use an intermediate approach by relating the macroscopic response of the material to simplified descriptions of the microscopic behavior. Macroscopic models therefore strike a balance between efficiency, accuracy, and predictive capability. The classical macroscopic models are the Preisach model [4], the Globus model [5], the Jiles-Atherton model [6], and the Stoner-Wohlfarth model [7]. Liorzou et al. [8] compare these models in detail.

Armstrong [9] proposed an incremental model for magnetoelastic hysteresis which calculates the bulk magnetization and magnetostriction as the expected values of a large collection of noninteracting magnetic moments. The probability density function is a Boltzmann distribution, where minimum energy orientations are more probable. The Armstrong model is computationally inefficient, as it searches for global energy minima. Atulasimha et al. [10] improved efficiency by only considering 98 fixed orientations. Evans and Dapino [11] proposed a discrete energy-averaged (DEA) model, which greatly improved efficiency while maintaining accuracy by solving for the local energy minimum about each of Galfenol's six easy crystallographic directions. The speed 
of this model is further increased by Chakrabarti [13], whose model is the starting point of our development.

The DEA model calculates the magnetization unit directions that minimize a Gibbs free energy defined locally about the six easy directions. However, the resulting minimization problem is not amenable to an explicit analytical solution. To alleviate this shortcoming, Evans and Dapino [11] linearized the normalization constraint about each easy direction, which is valid for small rotations of the magnetic moments about the easy directions. This novel treatment resulted in an explicit approximate solution, whose error is minimal since moments that have rotated far from the easy axes are more energetic, and thus less probable [12]. Nonetheless, the approximate magnetization directions may significantly violate the unity norm constraint for large inputs. Post-normalization of the directions was proposed by Chakrabarti and Dapino [14], but the resulting directions can still deviate from the true energy minima for generic 3D inputs. Additionally, this solution procedure is prone to singularities, which could burden the computation, especially when the method is integrated into finite element solvers.

The primary objective of this paper is to develop a robust solution procedure for the DEA model that avoids singularities. First, we improve the DEA model by incorporating the more thorough expression for the magnetostriction in cubic ferromagnets and by formulating the model based on the average of hysteretic data, which precludes the need for additional anhysteretic data. Then, an analytical solution of the resulting model is derived that exactly solves the constrained energy minimization. Depending on the dimension of the application, the solution procedure offers multiple solutions for each easy direction, which allows it to circumvent singularities and completely reveal the material behavior for arbitrary magnetic field and stress inputs. By using the analytical solution, the model is significantly reduced for $2 \mathrm{D}$ and $1 \mathrm{D}$ applications. A novel parameter optimization routine is developed, which decouples the model parameters into two sets. One set is quickly calculated through a preprocessing step, while the other is determined through a sophisticated constrained minimization. It is shown that the increased robustness of the proposed model comes at no expense for $1 \mathrm{D}$ applications, but requires twice the computation time for generic 3D applications. The model is validated through comparison with existing measurements and the former model.

The rest of the paper is organized as follows. A brief review of the DEA model and the existing approximate solution is shown in Section 2. Section 3.1 presents a reformulation of the DEA model and the analytical solution procedure. Section 4 gives the reduced formulation of the model for 2D and 1D applications. Parameter optimization is discussed in Section 5, which is followed by model 
validation and the conclusions.

\section{Review of calculation of $3 \mathrm{D}$ magnetostriction $(\lambda)$ and magnetization $(\mathrm{M})$}

Evans and Dapino [11] proposed a discrete energy-averaged (DEA) model that computes the magnetization directions of mesoscopic magnetic domains by minimizing the Gibbs free energy that is defined locally about each easy crystallographic direction. The Gibbs free energy in the vicinity of the $k^{\text {th }}$ easy direction is composed of magnetocrystalline (anisotropy), magnetoelastic (magnetomechanical coupling), and magnetic field (Zeeman) energies. The minimization procedure applied to Evans and Dapino's formulation requires as many matrix inversions as the number of easy directions. To reduce the number of matrix inversions to one, Chakrabarti [13] slightly modified the anisotropy energy. Accordingly, the Gibbs free energy can be written in matrix notation as

$$
\stackrel{k}{G}=\frac{1}{2} \stackrel{k}{\mathbf{m}} \cdot \overline{\mathbf{K}} \stackrel{k}{\mathbf{m}}-\stackrel{k}{\mathbf{b}} \cdot \stackrel{k}{\mathbf{m}}+\frac{1}{2} K+\stackrel{k}{K_{0}},
$$

where $K$ and $\stackrel{k}{K}$ are anisotropy energy constants; $\stackrel{k}{\mathbf{m}}=\left[\stackrel{k}{m}{ }_{1} ; \stackrel{k}{m_{2}} ; \stackrel{k}{m_{3}}\right]$ is the magnetization direction having unit magnitude; $k$ takes values $\pm 1, \ldots, \pm \frac{r}{2}$; and $r$ is the number of easy crystallographic directions (the $\langle 100\rangle$ family of six directions for Galfenol). The magnetic stiffness matrix $\overline{\mathbf{K}}$ and magnetic force vector $\mathbf{b}$ along the $k^{\text {th }}$ easy direction are given by

$$
\overline{\mathbf{K}}=K \mathbf{I}-3\left[\begin{array}{ccc}
\lambda_{100} T_{1} & \lambda_{111} T_{4} & \lambda_{111} T_{6} \\
\lambda_{111} T_{4} & \lambda_{100} T_{2} & \lambda_{111} T_{5} \\
\lambda_{111} T_{6} & \lambda_{111} T_{5} & \lambda_{100} T_{3}
\end{array}\right]
$$

and

$$
\stackrel{k}{\mathbf{b}}=K \stackrel{k}{\mathbf{c}}+\mu_{0} M_{s} \mathbf{H}
$$

respectively, where $\mathbf{I}$ is the $3 \times 3$ identity matrix; $\lambda_{100}$ and $\lambda_{111}$ are magnetostriction constants; $\mu_{0}$ and $M_{s}$ are, respectively, the vacuum permeability and saturation magnetization; $\mathbf{H}=\left[H_{1} ; H_{2} ; H_{3}\right]$ is the magnetic field vector; and $\mathbf{T}=\left[T_{1} ; T_{2} ; T_{3} ; T_{4} ; T_{5} ; T_{6}\right]$ stands for the stress tensor written in contracted vector notation, where $T_{1}=T_{11}, T_{2}=T_{22}, T_{3}=T_{33}, T_{4}=T_{12}, T_{5}=T_{23}$, and $T_{6}=T_{13}$.

The macroscopic 3D magnetostriction $\boldsymbol{\lambda}$ and magnetization $\mathbf{M}$ are defined as weighted sums of the response due to the $r$ minimum energy directions,

$$
\boldsymbol{\lambda} \triangleq \overline{\boldsymbol{\lambda}}=\sum_{k= \pm 1}^{ \pm r / 2} \stackrel{k}{\xi}_{a n} \lambda
$$




$$
\mathbf{M} \triangleq \overline{\mathbf{M}}=M_{s} \sum_{k= \pm 1}^{ \pm r / 2} \stackrel{k}{\xi}_{a n} \stackrel{k}{\mathbf{m}}
$$

where $\stackrel{k}{\xi}_{\text {an }}$ and $\stackrel{k}{\boldsymbol{\lambda}}$ denote, respectively, the bulk anhysteretic volume fraction and the magnetostriction tensor written in vector notation for the $k^{\text {th }}$ domain. Letting $\Omega$ be a smoothing factor, the former is calculated as a Boltzman-type, energy-weighted average as

$$
\stackrel{k}{\xi}_{a n}=\exp \left(-\frac{k}{\Omega}\right) \sum_{j= \pm 1}^{ \pm r / 2} \exp \left(\frac{j}{G}\right),
$$

and the magnetostriction in tensor notation is given as

$$
\begin{aligned}
& {\stackrel{k}{\boldsymbol{\lambda}_{u u}}}^{=}=\frac{3}{2} \lambda_{100} \stackrel{k}{m}_{u}^{2} \\
& \boldsymbol{\lambda}_{u v}=3 \lambda_{111} \stackrel{k}{m_{u}} \stackrel{k}{m}_{v}, \quad u \neq v
\end{aligned}
$$

\subsection{Calculation of $\stackrel{k}{m}$ (approximate solution)}

The application of an external magnetic field or stress changes the energy surface in the vicinity of each easy crystallographic direction, which may shift the $k^{t h}$ minimum energy direction away from the $k^{\text {th }}$ easy direction. Thus, the unit magnitude, minimum energy directions $\mathbf{m}$ are not known a priori for each easy direction, and must be calculated by minimizing the local free energies, eq. (1), before evaluating the volume fractions, eq. (14). Following Evans and Dapino [11], the constrained minimization is formulated as the following inhomogeneous eigenvalue problem through the application of the Lagrange multipliers method,

$$
\begin{array}{r}
(\overline{\mathbf{K}}-\stackrel{k}{\gamma} \mathbf{I}) \stackrel{k}{\mathbf{m}}=\stackrel{k}{\mathbf{b}}, \\
\stackrel{k}{\mathbf{m}} \cdot \stackrel{k}{\mathbf{m}}=1
\end{array}
$$

where $\stackrel{k}{\gamma}$ is the unknown Lagrange multiplier corresponding to the $k^{t h}$ minimum energy direction. In the absence of an explicit, analytical solution to the foregoing system of equations, Evans and Dapino [11] relaxed the normalization constraint through the approximation $\mathbf{m} \cdot \mathbf{m} \approx \mathbf{c} \cdot \mathbf{m}=1$ for each easy direction. As a result, the following explicit, approximate solution was reported:

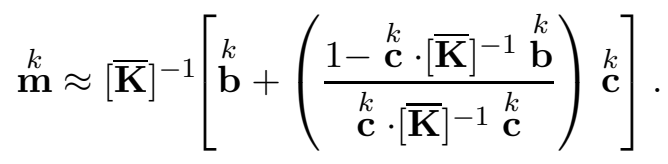




\subsection{Magnetomechanical hysteresis}

Evans and Dapino [11] presented an incremental form of hysteresis based upon an evolution equation for the volume fractions,

$$
\Delta \stackrel{k}{\xi}(i)=(1-c) \Delta \stackrel{k}{\xi}_{\text {irr }}(i)+c \Delta \stackrel{k}{\xi} \text { an }(i)
$$

where $i$ is the increment index, $c$ is a dimensionless constant quantifying the reversible processes during domain wall motion, and finally the change in the irreversible volume fractions for 3D stress and field inputs is defined as

$$
\begin{aligned}
& \Delta \stackrel{k}{\xi}_{\text {irr }}(i)= \\
& \left.\frac{\zeta}{k_{p}} \stackrel{k}{\xi}_{\text {an }}(i)-\stackrel{k}{\xi}_{\text {irr }}(i-1)\right)\left[\mu_{0} M_{s} \sum_{p=1}^{3}\left|\Delta H_{p}(i)\right|+(3 / 2) \lambda_{100} \sum_{p=1}^{3}\left|\Delta T_{p}(i)\right|+3 \lambda_{111} \sum_{q=4}^{6}\left|\Delta T_{q}(i)\right|\right],
\end{aligned}
$$

where $\zeta$ is a binary number for avoiding a nonphysical negative susceptibility, and $k_{p}$ is a pinning site density constant that characterizes the energy loss associated with domain wall rotation. Note that this hysteresis model is not self-starting, because the initial irreversible volume fractions are undefined. However, it is common practice to assume that $\xi_{i r r}(0)=0$.

\section{Reformulation of the DEA Model with Exact Solution Procedure}

The ultimate goal of the paper is to model Galfenol's hysteretic behavior. Therefore, we reformulate the DEA model based on the average of hysteretic data, as opposed to anhysteretic data, which would require additional measurements for parameter optimization and validation. In

essence, we employ the averaged hysteretic volume fractions, i.e. $\bar{\xi}_{\text {hys }}$, which correspond to the median curves obtained from averaging the hysteretic measurement data. Thus, every occurrence of $\xi_{a n}$ in the previous model is replaced by $\bar{\xi}_{h y s}$ in the new model. To be precise, eqs. (4)-(6), which define the nonhysteretic model, become

$$
\begin{gathered}
\boldsymbol{\lambda} \triangleq \overline{\boldsymbol{\lambda}}=\sum_{k= \pm 1}^{ \pm r / 2} \frac{k}{\bar{\xi}_{h y s}}{ }^{k}, \\
\mathbf{M} \triangleq \overline{\mathbf{M}}=M_{s} \sum_{k= \pm 1}^{ \pm r / 2} \frac{k}{\bar{\xi}_{h y s}} \stackrel{k}{\mathbf{m}},
\end{gathered}
$$




$$
\frac{k}{\xi}_{\text {hys }}=\exp \left(-\frac{k}{\Omega}\right) \sum_{j= \pm 1}^{ \pm r / 2} \exp \left(\frac{\stackrel{j}{G}}{\Omega}\right),
$$

where the parameters in the Gibbs free energy are optimized against the averaged hysteretic data; and finally eqs. (10) and (11), which constitute the hysteretic model, become

$$
\Delta \stackrel{k}{\xi}(i)=(1-c) \Delta \stackrel{k}{\xi}_{\text {irr }}(i)+c \Delta \stackrel{k}{\xi}_{\text {hys }}(i)
$$

$$
\begin{aligned}
& \Delta \stackrel{k}{\xi}_{\text {irr }}(i)= \\
& \frac{\zeta}{k_{p}}\left(\frac{k}{\bar{\xi}_{\text {hys }}}(i)-\stackrel{k}{\xi}_{\text {irr }}(i-1)\right)\left[\mu_{0} M_{s} \sum_{p=1}^{3}\left|\Delta H_{p}(i)\right|+(3 / 2) \lambda_{100} \sum_{p=1}^{3}\left|\Delta T_{p}(i)\right|+3 \lambda_{111} \sum_{q=4}^{6}\left|\Delta T_{q}(i)\right|\right],
\end{aligned}
$$

We also reformulate the magnetoelastic energy. Evans and Dapino [11] used the tensor-valued magnetostriction, eq. (7), given by Engdahl [15]. Instead, we use the more thorough formulation of Kittel [16] with a constant term not present in the book by Engdahl. Kittel's expressions are given in component tensor notation as

$$
\begin{aligned}
& {\stackrel{k}{\lambda_{u u}}=\frac{3}{2} \lambda_{100}\left(m_{u}^{k}\right.}_{2}^{2}-\overbrace{\frac{c_{12}}{c_{11}+2 c_{12}}}^{\triangleq c_{0}}, \\
& \stackrel{k}{\boldsymbol{\lambda}_{u v}}=3 \lambda_{111} \stackrel{k}{m_{u} m_{v}^{k}}, \quad u \neq v,
\end{aligned}
$$

where $u, v \in\{1,2,3\}$, and $c_{11}$ and $c_{12}$ are two of the three independent elastic moduli for crystals with cubic symmetry. Relative to Engdahl's equation used in the prior work, employment of eq. (17) provides a better fit of the model to experimental data for the sensing case, as detailed in Subsection 6.1.

\subsection{Calculation of $\stackrel{k}{m}$ (exact solution)}

The approximate solution, eq. (9), has the advantage of simplicity and explicitness. However, the approximate magnetization direction obtained from eq. (9) violates the unity norm constraint when the material is saturated by large magnetic fields or stresses. Referring to eq. (5), this results in a nonphysical magnetization (i.e., larger than the saturation magnetization $M_{s}$. One way to circumvent this issue is to normalize the approximate solution following its calculation[11, 13]. 
Although this might seem effective, the solution does not truly reflect the genuine solution of the system of eqs. (20). Additionally, the approximate method fails to provide a solution when the matrix $\overline{\mathbf{K}}$ becomes singular. For a planar stress state, i.e. $T_{3}=T_{5}=T_{6}=0$, this singularity occurs on the surface depicted in Figure 1, where the determinant sub-locus of the matrix $\overline{\mathbf{K}}$ is shown. An ill-conditioned matrix $\overline{\mathbf{K}}$ can also cause numerical difficulties, expanding the failure surface into a volume. Although planar stress is a special case, it is apparent that the singularity zone enlarges when the stress state is generic. The failure zone of such a case has too many dimensions to show graphically, but it is governed by the determinant constraint $|\overline{\mathbf{K}}|<\epsilon$, where $\epsilon$ is a small number. The need for a more robust model is further motivated considering that this constitutive model has been integrated into finite element models for the simulation of Galfenol-based systems [14, 17]. The utility of such finite element models for use by non-experts is hampered if the model does not incorporate a black-box constitutive model that is valid for all inputs.

(a)

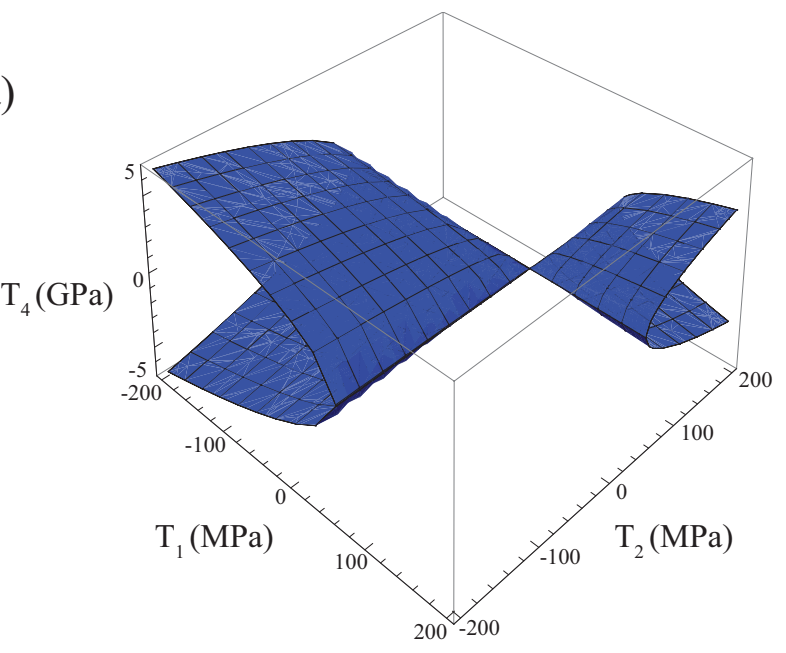

(b)

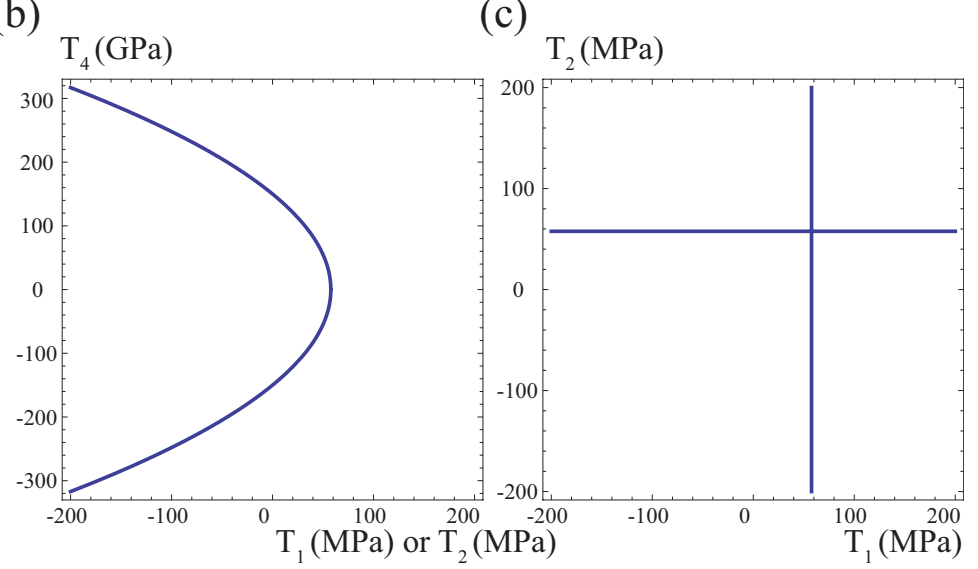

Figure 1: (a) The determinant sub-locus of the matrix $\overline{\mathbf{K}}$ corresponding to planar stress for $K=3 \times 10^{4}, 3 \lambda_{100}=$ $520 \times 10^{-6}$, and $3 \lambda_{111}=-20 \times 10^{-6}$, (b) slice plot for $T_{1}=0$ or $T_{2}=0$, and (c) slice plot for $T_{4}=0$. 
Before deriving the exact solution procedure, we simplified the Gibbs free energy, eq. (1), as (see Appendix)

$$
\stackrel{k}{G}=\frac{1}{2} \stackrel{k}{\mathbf{m}} \cdot \mathbf{K} \stackrel{k}{\mathbf{m}}-\stackrel{k}{\mathbf{b}} \cdot \stackrel{k}{\mathbf{m}}+\stackrel{k}{K}{ }_{0}=\frac{1}{2}(\stackrel{k}{\gamma}-\stackrel{k}{\mathbf{b}} \cdot \stackrel{k}{\mathbf{m}})+\stackrel{k}{K_{0}},
$$

where the simplified magnetic stiffness matrix is,

$$
\mathbf{K}=-3\left[\begin{array}{ccc}
\lambda_{100} T_{1} & \lambda_{111} T_{4} & \lambda_{111} T_{6} \\
\lambda_{111} T_{4} & \lambda_{100} T_{2} & \lambda_{111} T_{5} \\
\lambda_{111} T_{6} & \lambda_{111} T_{5} & \lambda_{100} T_{3}
\end{array}\right]
$$

The energy minimization problem becomes

$$
\begin{array}{r}
(\mathbf{K}-\stackrel{k}{\gamma} \mathbf{I}) \stackrel{k}{\mathbf{m}}=\stackrel{k}{\mathbf{b}}, \\
\stackrel{k}{\mathbf{m}} \cdot \stackrel{k}{\mathbf{m}}=1
\end{array}
$$

Such simplifications reduce computation time and are crucial when the model is integrated into finite element constructs, which evaluate the model many times for each solution step. To make the model more robust, an exact solution to the system given by eq. (20a) and (20b) is derived as follows. By employing the eigenvalue decomposition, eq. (20a) can be rewritten as

$$
\begin{aligned}
\stackrel{k}{\mathbf{b}} & =(\mathbf{K}-\stackrel{k}{\gamma} \mathbf{I}) \stackrel{k}{\mathbf{m}}=\left(\mathbf{Q} \mathbf{\Lambda} \mathbf{Q}^{\mathrm{T}}-\stackrel{k}{\gamma} \mathbf{I}\right) \stackrel{k}{\mathbf{m}} \\
& =\left(\mathbf{Q} \mathbf{\Lambda} \mathbf{Q}^{\mathrm{T}}-\stackrel{k}{\gamma} \mathbf{Q} \mathbf{Q}^{\mathrm{T}}\right) \stackrel{k}{\mathbf{m}} \\
& =\mathbf{Q}(\mathbf{\Lambda}-\stackrel{k}{\gamma} \mathbf{I}) \mathbf{Q}^{\mathrm{T}} \stackrel{k}{\mathbf{m}},
\end{aligned}
$$

where $\mathbf{Q}$ is an orthogonal matrix containing the eigenvectors of $\mathbf{K}$, and $\boldsymbol{\Lambda}$ is a diagonal matrix composed of the corresponding eigenvalues, i.e. $\lambda_{1-3}$, of $\mathbf{K}$. Solving eq. (21) for $\stackrel{k}{\mathbf{m}}$ and substituting it into eq. (20b) one gets

$$
{ }^{k}{ }^{\mathrm{T}} \mathbf{Q}(\boldsymbol{\Lambda}-\stackrel{k}{\gamma} \mathbf{I})^{-2} \mathbf{Q}^{\mathrm{T}} \stackrel{k}{\mathbf{b}}=1
$$

The matrix inversion required in the foregoing equation is easily avoided by using the simplification 


$$
(\boldsymbol{\Lambda}-\stackrel{k}{\gamma} \mathbf{I})^{-n}=\left[\begin{array}{ccc}
\frac{1}{\left(\lambda_{1}-\gamma\right)^{n}} & 0 & 0 \\
0 & \frac{1}{\left(\lambda_{2}-\gamma\right)^{n}} & 0 \\
0 & 0 & \frac{1}{\left(\lambda_{3}-\frac{k}{\gamma}\right)^{n}}
\end{array}\right], \quad n \in \mathbb{N}
$$

When expanded, eq. (22) reduces to the following sixth order polynomial:

$$
\begin{array}{r}
\frac{k}{\gamma}+2\left(\bar{\lambda}_{2}+\bar{\lambda}_{3}\right) \frac{k}{\gamma} 5+\left(\bar{\lambda}_{2}^{2}+4 \bar{\lambda}_{2} \bar{\lambda}_{3}+\bar{\lambda}_{3}^{2}-\bar{Q}_{1}-\bar{Q}_{2}-\bar{Q}_{3}\right) \frac{k}{\gamma}+2\left(\bar{\lambda}_{2}^{2} \bar{\lambda}_{3}+\bar{\lambda}_{2} \bar{\lambda}_{3}^{2}-\bar{\lambda}_{2} \bar{Q}_{1}\right. \\
\left.-\bar{\lambda}_{3} \bar{Q}_{1}-\bar{\lambda}_{3} \bar{Q}_{2}-\bar{\lambda}_{2} \bar{Q}_{3}\right) \frac{k}{\gamma}{ }^{3}+\left(\bar{\lambda}_{2}^{2} \bar{\lambda}_{3}^{2}-\bar{\lambda}_{2}^{2} \bar{Q}_{1}-4 \bar{\lambda}_{2} \bar{\lambda}_{3} \bar{Q}_{1}-\bar{\lambda}_{3}^{2} \bar{Q}_{1}-\bar{\lambda}_{3}^{2} \bar{Q}_{2}-\bar{\lambda}_{2}^{2} \bar{Q}_{3}\right) \frac{k}{\gamma}{ }^{2} \\
-2 \bar{\lambda}_{2} \bar{\lambda}_{3} \bar{Q}_{1}\left(\bar{\lambda}_{2}+\bar{\lambda}_{3}\right) \frac{k}{\gamma}-\bar{\lambda}_{2}^{2} \bar{\lambda}_{3}^{2} \bar{Q}_{1}=0
\end{array}
$$

where $\frac{k}{\gamma}=\lambda_{1}-\stackrel{k}{\gamma}, \bar{\lambda}_{2}=\lambda_{2}-\lambda_{1}, \bar{\lambda}_{3}=\lambda_{3}-\lambda_{1}$, and $\left[\sqrt{\bar{Q}_{1}} ; \sqrt{\bar{Q}_{2}} ; \sqrt{\bar{Q}_{3}}\right]=\mathbf{Q}^{T} \stackrel{k}{\mathbf{b}}$. For the $k^{\text {th }}$ easy direction, this polynomial can be easily solved to obtain the entire solution set of $\gamma$, which is consequently used to calculate a set of magnetization directions after solving eq. (21) for $\mathbf{m}$ as

$$
\stackrel{k}{\mathbf{m}}=\mathbf{Q}(\boldsymbol{\Lambda}-\stackrel{k}{\gamma} \mathbf{I})^{-1} \mathbf{Q}^{T} \stackrel{k}{\mathbf{b}}
$$

Again, the matrix inversion is easily avoided using eq. (23).

\subsubsection{Multiplicity of the solution set for $\stackrel{k}{m}$}

Due to the dimensionality of the problem and the unity norm constraint (eq. (20b)), eq. (24) is a sixth order polynomial, giving rise to six possible solutions for $\mathbf{m}$ for each $k$. The complex solutions of eq. (24) can be neglected. Since the expression for $G$ for each $k$ is only valid in the vicinity of the $k^{\text {th }}$ easy magnetization direction, the direction solutions oriented sufficiently far from the corresponding easy axis can also be ignored. For example, Figure 2 shows the volume of valid magnetization directions about $\stackrel{-2}{\mathbf{c}}=[0 ;-1 ; 0]$. The remaining solutions of eq. (24) correspond to valid minima of the local energy function $G$ for each $k$. Figure 3 depicts a surface plot of Gibbs free energy (scaled down by $K$ ), showing the real, exact solutions, eq. (25), and the approximate solution (eq. (9)) of $\mathbf{m}$ for each $k$. Clearly, the approximate solution does not truly correspond to the Gibbs free energy minimizer.

The proposed modeling framework has the capability of considering all valid solutions of $\mathbf{m}$ for each $k$; however, the volume fraction of magnetic domains oriented along each minima depends upon the history of stress and magnetic field application as well as thermal activation, which energizes 


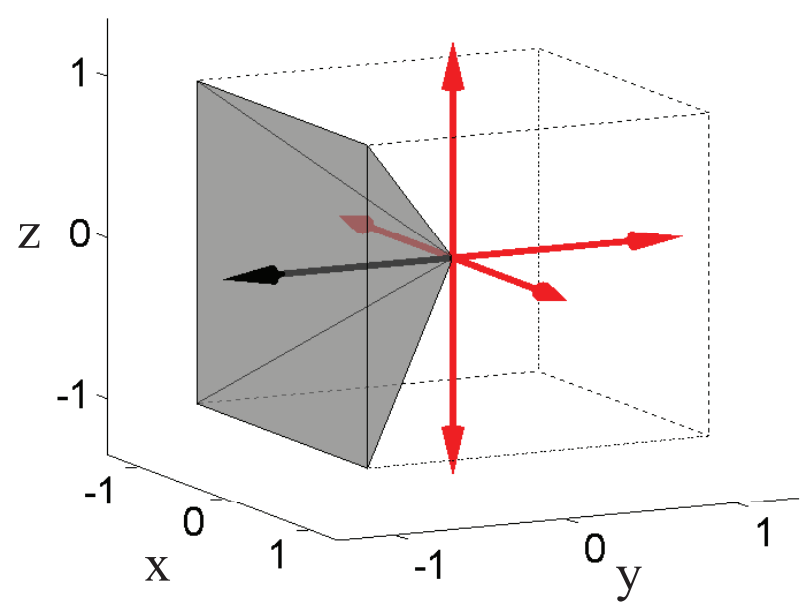

Figure 2: Volume of valid magnetization directions (shaded region) for $\mathbf{m}_{\mathbf{m}}^{2}$ in the vicinity of $\mathbf{c}^{2}=[0 ;-1 ; 0]$ (black arrow), shown in relation to the 5 other easy magnetization directions (red arrows) for a material with cubic anisotropy and $\stackrel{k}{K}>0$.

magnetic moments allowing them to overcome the energy barriers between energy minima. Thus, if more than one magnetization direction for each easy direction is considered, the energy weighting expression (eq. (14)) should be modified to incorporate these effects, while ensuring that multiple minima about a single easy direction are not excessively weighted. Following the approach of Evans and Dapino [11], this work strikes a balance between accuracy and efficiency by considering only six magnetization directions. For each easy direction, the solutions for $\mathbf{m}$ are selected using the criteria that each minimizes most the Gibbs free energy within their respective volume of valid magnetization directions.

\section{Simplification of the Model for Lower Dimensional Applications}

Galfenol can be utilized in complex 3D systems, because it can withstand 3D stresses. However, not all applications require Galfenol's 3D capability. For such applications, lower dimensional models are sufficient or are useful for preliminary analysis of the system. In fact, many experiments are conducted on magnetostrictive rods, which are exposed to an axial magnetic field and stress, thus warranting even a 1D formulation of the proposed model.

While the exact solution procedure presented in Section 3 is general and is valid for arbitrary applications, a special magnetic field or stress state may reduce the number of locally-minimum energy directions. For such cases, eq. (24) degenerates into lower order polynomials, which can be solved more efficiently. Two cases are considered: 2D (plane stress) and 1D. Note that the over 


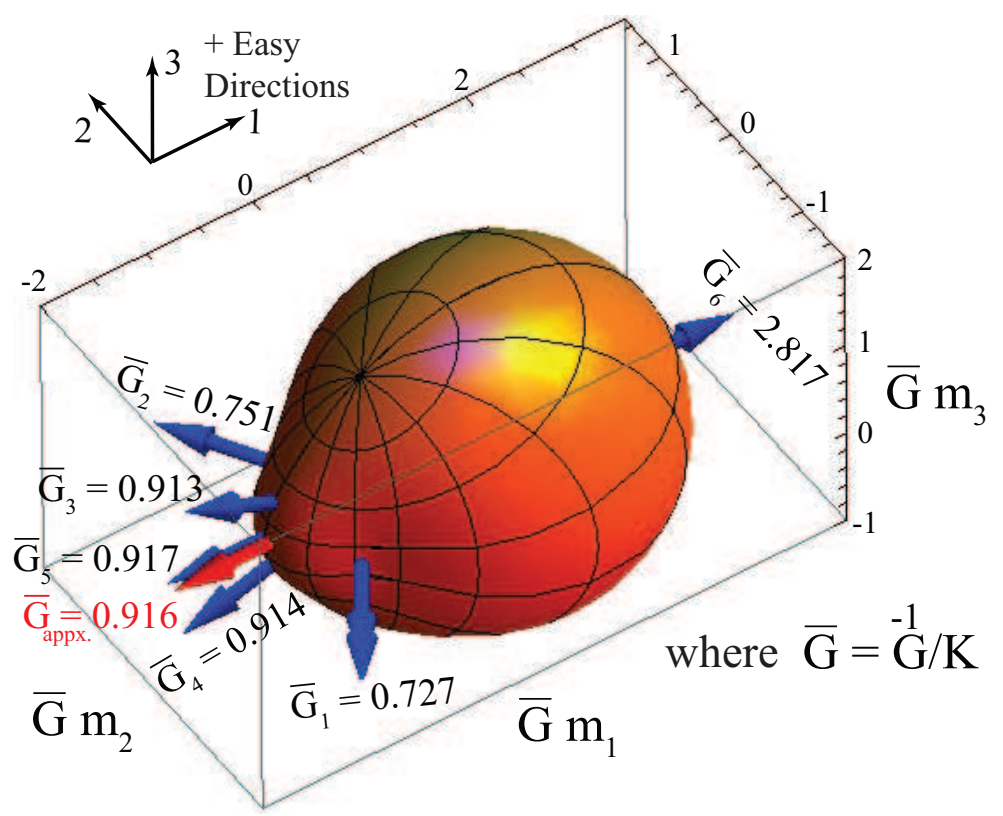

Figure 3: The cartesian surface plot of Gibbs free energy (eq. (1)), scaled down by $K$, relative to the second easy direction $\mathbf{c}^{-1}$ with $K=30\left(\mathrm{~kJ} / \mathrm{m}^{3}\right), K_{100}=0\left(\mathrm{~kJ} / \mathrm{m}^{3}\right), 3 \lambda_{100}=520(\mathrm{ppm})$ and $3 \lambda_{111}=-20(\mathrm{ppm})$ for $\mathbf{H}=[1 ; 0 ; 0](\mathrm{kA} / \mathrm{m})$ and $\mathbf{T}=-[100 ; 0 ; 40 ; 40 ; 40 ; 0](\mathrm{MPa}) ;$ real solutions of eq. (25) shown by blue arrows (orientation most minimizing energy by a double-headed arrow); approximate solution (eq. (9)) shown by a red arrow.

index $k$ is dropped within subsections that specify its value to improve clarity.

\subsection{D Applications}

Consider a state of plane stress and planar magnetic field (i.e., $H_{3}=T_{3}=T_{5}=T_{6}=0$ ). For this case, eq. (24) still has six solutions for \pm 3 easy directions. However, a simplified approach can be used for the remaining easy directions.

\subsection{1. $k= \pm 1, \pm 2$}

For the easy crystallographic directions $\pm\langle 100\rangle$ and $\pm\langle 200\rangle$, the magnetic force vector (eq. (3)) has one zero component, namely $b_{3}=0$. From eqs. (20), this results in a planar magnetization direction (i.e., $m_{3}=0$ ) since $\gamma$ cannot equal 0 . The remaining direction components are

$$
m_{2}=\frac{K_{12}\left(2 m_{1}^{2}-1\right)-b_{2} m_{1}}{\hat{K}_{12} m_{1}-b_{1}}= \pm \sqrt{1-m_{1}^{2}}
$$

where $K_{11}=-\frac{3}{2} \lambda_{100} T_{1}, K_{12}=-\frac{3}{2} \lambda_{111} T_{4}$, and $\hat{K}_{12}=-\frac{3}{2} \lambda_{100}\left(T_{1}-T_{2}\right)$. Substituting the foregoing result into eq. (20b) gives the fourth order polynomial 


$$
\begin{aligned}
&\left(\hat{K}_{12}^{2}+4 K_{12}^{2}\right) m_{1}^{4}-2\left(b_{1} \hat{K}_{12}+2 b_{2} K_{12}\right) m_{1}^{3}+\left(b_{1}^{2}+b_{2}^{2}-\hat{K}_{12}^{2}-4 K_{12}^{2}\right) m_{1}^{2} \\
&+2\left(b_{1} \hat{K}_{12}+b_{2} K_{12}\right) m_{1}+K_{12}^{2}-b_{1}^{2}=0 .
\end{aligned}
$$

\subsection{D Applications}

Consider 1D loading along $\pm\langle 100\rangle$, for which the only nonzero stress and field components are $T_{1}$ and $H_{1}$.

\subsection{1. $k= \pm 1$}

For the easy crystallographic directions $\pm\langle 100\rangle$, the magnetic force vector (eq. (3)) has components $b_{2}=b_{3}=0$, and the system of eqs. (20) has the explicit solutions $\stackrel{1}{\mathbf{m}}=\stackrel{1}{\mathbf{c}}$ and $\stackrel{-1}{\mathbf{m}}=\stackrel{-1}{\mathbf{c}}$ since $\gamma$ cannot equal 0 .

\subsection{2. $k= \pm 2, \pm 3$}

Magnetic domains tend to orient parallel to magnetic fields and tensile stresses, and perpendicular to compressive stresses. For a 1D application, there is no driving force to rotate the basal plane magnetization directions (i.e., those of $k= \pm 2, \pm 3$ ) about the loading axis $\pm\langle 100\rangle$. The basal plane directions, therefore, respond equivalently to axial loading; thus, it is sufficient to consider only the case $k=2$. Solving eq. (20a) for $m_{2}$ one gets

$$
m_{2}=\frac{b_{2} m_{1}}{b_{1}-K_{11} m_{1}}= \pm \sqrt{1-m_{1}^{2}}
$$

Substitution of the foregoing result into eq. (20b) gives

$$
K_{11}^{2} m_{1}^{4}-2 b_{1} K_{11} m_{1}^{3}+\left(b_{1}^{2}+b_{2}^{2}-K_{11}^{2}\right) m_{1}^{2}+2 b_{1} K_{11} m_{1}-b_{1}^{2}=0
$$

Unlike the exact solution procedure for 3D inputs, the reduced 1D and 2D models do not require a solution for the intermediate variable $\gamma$; rather, the exact minimum energy directions are directly found by solving the corresponding fourth order polynomials. In particular, due to the equivalence of the four basal plane directions for 1D applications, eq. (29) must be solved only once. As detailed later, the 1D simplification is over twice as efficient as the proposed 3D model. 


\section{Parameter Optimization}

The goal of the parameter optimization procedure is to identify the constant model parameters $\left\{M_{s}, E, \lambda_{100}, \lambda_{111}, c_{0}, K, K_{0}, \Omega\right\}$ that provide the best fit of the model to experimental data. With optimized parameters, the model should adequately calculate the magnetization and magnetostriction response for arbitrary input sets (i.e., interpolated within or extrapolated outside the inputs used for experimental validation).

The optimization procedure presented below is dichotomous. First, averaged experimental data is found from 1D hysteretic measurements through an effective averaging technique. Second, a sophisticated objective error function relating the family of simulated responses and the family of averaged data is minimized to determine the optimal material parameters.

\subsection{Extracting the averaged responses from hysteretic measurements}

The aim is to average the upper and lower branches of hysteretic data. Chakrabarti [13] proposed a technique that averages in an incremental sense with a user-defined step size. However, even for a carefully chosen step size, some portions of the averaged curves obtained from this approach may go outside the two hysteretic curves, suggesting that the technique lacks robustness. To alleviate this shortcoming, the following two issues must be addressed. First, for sampled hysteretic actuation and sensing curves, the values of the independent variable for points on the upper and lower branches may not match. To alleviate this, each branch is fit with piecewise linear Hermite functions and resampled using the same discretization.

Second, the data in highly-sloped burst regions may need to be treated differently from that in the flat saturation regions. For the former, a major change is observed in the vertical direction, and thus the data should be averaged in the horizontal direction. For the latter, the opposite is true. Accordingly, this switch of the averaging direction is taken into account in the discretization procedure discussed above.

\subsection{Objective Error Function}

MATLAB's built-in constrained optimization function, fmincon, is used. This function needs an initial guess and bounds for each parameter, and a globally-defined, scalar objective function. For $c_{0}$ and $\Omega$, respectively, $10^{-5}$ and 1200 were used as start guesses. For the remaining initial guesses, the approximate analytical expressions developed by Chakrabarti [13] were utilized. Furthermore, the bounds for all parameters were selected to be $80 \%$ above and below the corresponding initial 
guesses, except the smoothing factor $\Omega$, for which a range from 0.01 to $3 \mathrm{~kJ} / \mathrm{m}^{3}$ was selected. Finally, the objective function is a weighted average error for an entire family of curves that is constructed as follows.

1. For the $i^{t h}$ curve of $N_{i}$ points in a data set, the modeling error is quantified as

$$
\operatorname{error}_{i}=\frac{1}{N_{i}} \sum_{j=1}^{N_{i}}\left|w_{i j} \frac{Y_{i j}-X_{i j}}{\operatorname{range}\left(X_{i}\right)}\right|^{n},
$$

where $Y_{i j}$ and $X_{i j}$ are the $j^{\text {th }}$ component of the $i^{\text {th }}$ calculated response and averaged data, respectively, which contain $N_{i}$ points; $\operatorname{range}\left(X_{i}\right)$ is the difference between the upper and lower bound for that curve; $w_{i j}$ is a weighting factor for each point; $n$ is a positive number, which when taken as 1 gives rise to a 1-norm, and when taken as 2 yields the square of the common Euclidean 2-norm.

2. The data is split into two sub-families: actuation and sensing. The mean error for each sub-family is obtained by averaging the individual errors within the sub-family.

3. The total error is the sum of the two sub-family errors.

Note that the error function definition (30) retains the main feature of that from Chakrabarti [13] in that the error is normalized with respect to the range of each curve, allowing for the safe combination of the magnetization and magnetostriction errors. In contrast, the new error function definition generalizes the former definion in two aspects. First, instead of taking $n$ to be $2, n$ can be any number, or can even be a parameter to be identified through optimization. Our experience suggests that $n=1$ provides good performance. Second, with the user-defined weighting factors $w_{i j}$, one can have more control over how much error is attributed to the different regions of the curves. In essence, the error observed in the burst regions dominates that of the saturation regions; thus the latter error, even if large, does not contribute much to the total error. Therefore, the parameters may be overly optimized for the burst regions. For such cases, a relatively large weighting factor is devised so that the resulting saturation error is comparable to that from the burst regions. We do not report values for the weighting factors, as their determination is done through trial and error and is dependent on the given data.

\subsection{Some Notes on the Optimization}

Finding the globally-optimal parameter set can be time consuming, because the parameter space has eight dimensions. To reduce the computational burden, the following notes may be considered. 
- When calculating the averaged data, one may discretize each curve using the same number of points. This allows for $N_{i}$ to be removed from the error function (eq. (30)).

- For ideal actuation measurements, the magnetization and magnetostriction curves are symmetric with respect to magnetic field. Thus, only half of the actuation data may need to be considered.

- The parameter set may be split into three sets: $\left\{M_{s}, E, \lambda_{100}\right\}$, and $\left\{c_{0}, K, K_{0}, \Omega\right\}$, and $\left\{\lambda_{111}\right\}$. The first set may be directly calculated from sensing and actuation responses (i.e., a preprocessing step), while the second set is found through optimization. For 1D measurements of a $\langle 100\rangle$-oriented sample, the third set cannot be calculated directly or through optimization, as the $1 \mathrm{D}$ model is independent of $\lambda_{111}$. In the absence of shear stresses, $\lambda_{111}$ can be arbitrarily set to zero. In other cases, this parameter must be taken from the literature or measured from $1 \mathrm{D}$ measurements of a $\langle 111\rangle$-oriented sample.

\section{Validation of the Model: Results and Discussion}

The model consists of two parts, i.e. the nonhysteretic and hysteretic parts. The former models the averaged hysteretic data, and is governed by eqs. (12)-(14) and (20), while the latter is built on the former, and is defined by eqs. (15) and (16). Validation of each part is discussed in the subsections below.

Due to the significant difficulty in obtaining 3D measurements, it is common practice to conduct 1D experiments, in which a magnetostrictive rod is exposed to load and magnetic field along its main axis, say $\pm\langle 100\rangle$ direction. As detailed above, a key benefit of the DEA model is that all but one parameter can be determined from 1D measurements. For validation, existing data obtained by Evans and Dapino [11] is used. The data was obtained using a textured, $\langle 100\rangle$-oriented $\mathrm{Fe}_{81.5} \mathrm{Ga}_{18.5}$ rod grown with the Free Stand Zone Melt method (FSZM) at Etrema Products Inc.

\subsection{Validation of the Nonhysteretic Model}

The constant parameters $\left\{M_{s}, E, \lambda_{100}, \lambda_{111}, c_{0}, K, K_{0}, \Omega\right\}$ must be identified to fully define the nonhysteretic model. The aforementioned measurement data is used to initiate the optimization routine discussed in Section 5. The optimized parameters are tabulated in Table 1. Figure 4 depicts the measured data, together with the extracted averaged curves along with the calculated nonhysteretic model. The figure clearly demonstrates the effectiveness of the averaging technique, 
and the close agreement between the nonhysteretic model and the averaged hysteretic data. Due to the decoupled optimization procedure (namely the calculated Young's modulus and saturation magnetization and magnetostriction), excellent fits are obtained in the saturation regimes.

Table 1: Optimized parameters for the nonhysteretic model.

\begin{tabular}{|c|cccc|}
\hline Par. & $M_{s}(\mathrm{kA} / \mathrm{m})$ & $E(\mathrm{GPa})$ & $\lambda_{100}(\mathrm{ppm})$ & $\lambda_{111}(\mathrm{ppm})$ \\
\hline Value & 1242.20 & 74.49 & 172.31 & - \\
\hline \hline Par. & $c_{0}$ & $K\left(\mathrm{~kJ} / \mathrm{m}^{3}\right)$ & $K_{100}\left(\mathrm{~J} / \mathrm{m}^{3}\right)$ & $\Omega(\mathrm{J})$ \\
\hline Value & 0.38 & 35.58 & 412.18 & 1330.00 \\
\hline
\end{tabular}

Common to all sub-figures of Figure 4, the model underestimates both magnetization and magnetostriction as the material leaves the burst region and approaches saturation. For instance, in sub-figure 4(a) for the $32.3 \mathrm{MPa}$ bias stress, the model starts to deviate from the averaged data near $7.3 \mathrm{kA} / \mathrm{m}$ and exhibits a maximum relative error of $19 \%$ at $8.2 \mathrm{kA} / \mathrm{m}$ before tending to zero as it saturates around $11 \mathrm{kA} / \mathrm{m}$. This consistent lack of agreement over all sub-figures suggests that some of the underlying physics are not captured by the local free energy formulation (eq. (18)). Improvements to this energy will be the subject of future research.

Table 2 compares the normalized error of the proposed model and the approximate model $[11,13]$ for magnetization and strain for actuation and sensing cases. The error was calculated according to subsection 5.2 with all the weighting factors set to one. The number of uniformly-distributed samples was increased until the error was unchanged. The table demonstrates that the total error of the new model is extremely low, i.e. only $9.3 \%$ of that of the approximate model.

Table 2: Comparison of the normalized error of the exact and approximate nonhysteretic models. Model parameters for the latter are obtained from Chakrabarti [13]: $M_{s}=1225.00(\mathrm{kA} / \mathrm{m}), E=75.31(\mathrm{GPa}), \lambda_{100}=166.31(\mathrm{ppm})$, $K=34.84\left(\mathrm{~kJ} / \mathrm{m}^{3}\right), K_{100}=498.00\left(\mathrm{~J} / \mathrm{m}^{3}\right)$, and $\Omega=1014.70(\mathrm{~J})$.

\begin{tabular}{|c|cccccc|c|}
\hline \multirow{2}{*}{ Model } & \multicolumn{3}{|c|}{ Actuation error } & & \multicolumn{2}{c|}{ Sensing error } & Total \\
\cline { 2 - 3 } & $M$ & $S$ & & $M$ & $S$ & error \\
\hline Exact & 0.04 & 0.06 & & 0.09 & 0.09 & 0.28 \\
\hline Approximate & 0.04 & 0.05 & & 0.08 & 2.84 & 3.01 \\
\hline
\end{tabular}

The major error of the approximate model for the sensing case originates from the magnetostriction equation given by eq. (7), which is replaced by eq. (17) in the proposed model. Figure 5 illustrates the effectiveness of incorporating the magnetostriction constant $c_{0}$ by comparing aver- 

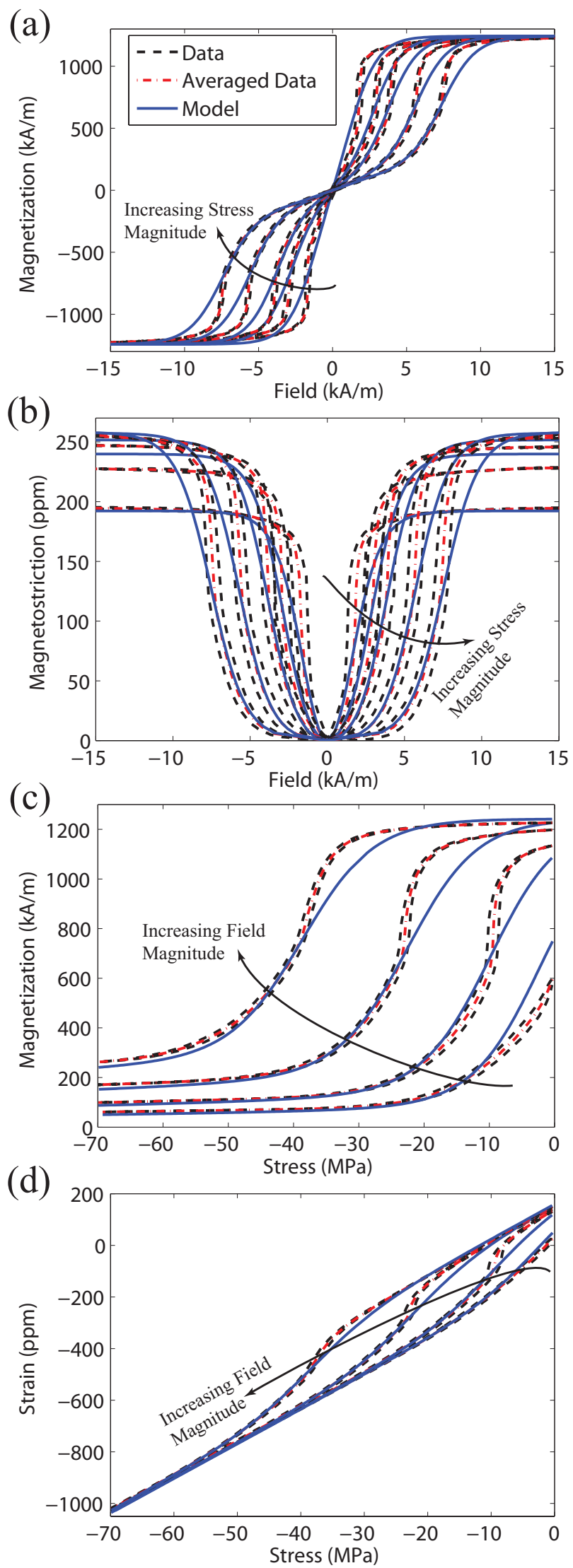

Figure 4: Comparison of averaged hysteretic measurements and nonhysteretic model for $\langle 100\rangle \mathrm{Fe}_{81.5} \mathrm{Ga}_{18.5}$ grown with FSZM at (a),(b) constant compressive stress valuessof 0.32, 8.00, 13.40, 23.10, and $32.30 \mathrm{MPa}$, and (c),(d) constant field values of $1.85,3.24,5.65$, and $8.88 \mathrm{kA} / \mathrm{m}$. Strain is the superposition of the mechanical strain (T/E) and the magnetostriction $(\lambda)$. 
(a)
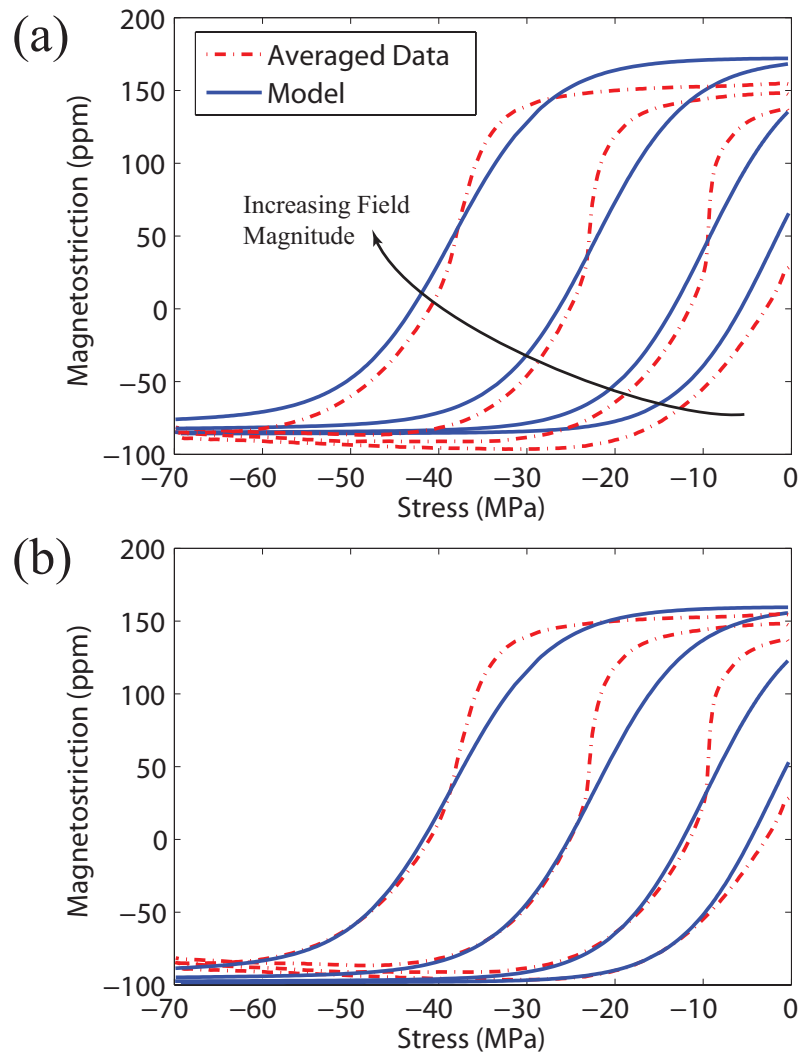

Figure 5: Magnetostriction calculated with (a) eq. (7) used in the prior work[11] and (b) eq. (17), and compared with the averaged hysteretic data at constant field values of $1.85,3.24,5.65$, and $8.88 \mathrm{kA} / \mathrm{m}$. For each case, material parameters are optimized for the full family of actuation and sensing measurements using identical objective error functions.

aged hysteretic data to simulations that use the current and previous magnetostriction expressions: eqs. (17) and (7), respectively. Since the stiffness moduli $c_{11}$ and $c_{12}$ are positive, $c_{0}$ is also positive. Consequently, the magnetostriction obtained from the prior formulation exceeds that from the current expression, which provides a better fit to the data. Furthermore, even though the constant $c_{0}$ appears to take part only in the calculation of the strain-stress sensing curves, the improved fitting accuracy, as shown in sub-figure 5(b), leads to a reduced total objective error value, thus improving the overall optimization procedure as demonstrated in Table 2.

\subsection{Computational Efficiency}

The analysis of an algorithm primarily concerns its computation time [18]. For modeling the macroscopic behavior of cubic magnetostrictive materials, the approximate formulation of the DEA model developed by Evans and Dapino [11] currently has the best combination of speed and accuracy. Since the same hysteresis model is used for the current (exact) and prior (approximate) works, the computational speed of the nonhysteretic models are compared. For the approximate 
model, optimized material parameters were obtained from Chakrabarti [13], while for the exact model, the optimized properties given in Table 1 were used.

To incorporate any effect of the input parameters on computation time, the input parameters were varied for each execution using quasi-random sampling; thus, the expected execution time was determined by simulating each model a large number of times and dividing by the number of executions [18]. Components of the input were sampled from the nine-dimensional parameter space (three for $\mathbf{H}$, and six for $\mathbf{T}$ ) using a Sobol' quasi-random (QR) sequence [19], which are commonly used in sensitivity analysis [20]. QR sequences generate points in the unit hypercube more uniformly than sequences of random numbers, thereby minimizing the number of samples required to form a representative sample of the parameter space. The Sobol' QR sequence is scaled such that $-70 \leq H_{j} \leq 70 \mathrm{kA} / \mathrm{m}$ and $-250 \leq T_{i} \leq 250 \mathrm{MPa}$, with the constraint that if a sampled stress state corresponds to a von Mises stress larger than $400 \mathrm{MPa}$, it is discarded.

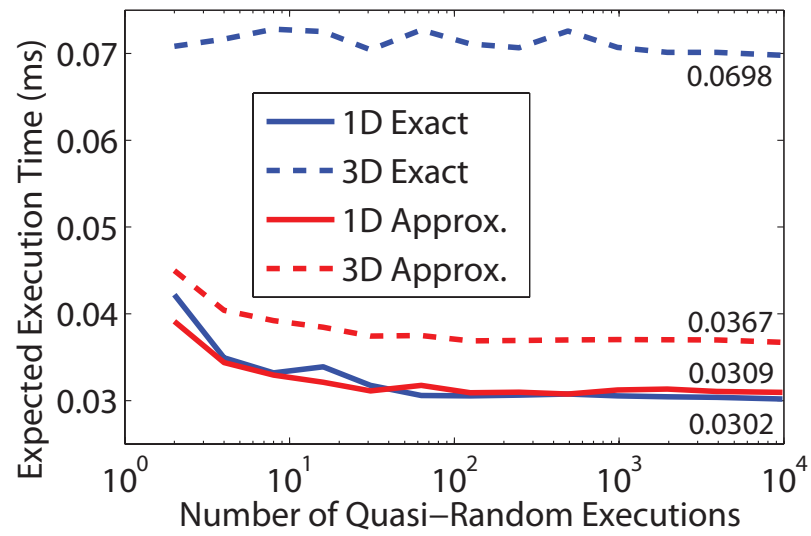

Figure 6: Expected execution time (averaged over 2 computers) of the exact and approximate constitutive models (coded as compiled MATLAB functions).

The models were coded as compiled MATLAB functions and executed on two computers. Since the performance of the $2 \mathrm{D}$ version of the exact model is bounded by that of the $1 \mathrm{D}$ and $3 \mathrm{D}$ versions, only the averaged results for $1 \mathrm{D}$ and $3 \mathrm{D}$ formulations of the models are shown in Figure 6 . While the exact model is slightly faster than the approximate model for 1D inputs, it is slower than the approximate model for 3D inputs, requiring about 1.9 times the CPU time. This is to be expected, due to the multiplicity of the solution set for $\mathbf{m}$, which requires more computation. Nonetheless, the exact model is more accurate and defined for all inputs, which is crucial for implementation in black-box finite element models. Additionally, a preliminary investigation of the material Jacobian derived from the exact model reveals that material derivatives are significantly simplified relative to 
those derived from the approximate model. This suggests that the exact model may be faster than the approximate model for 3D system-level modeling, which requires the Jacobian for inversion of the constitutive model and calculation of Galfenol's stiffness and sensitivities. This last feature with relevant applications will be discussed in a future paper.

\subsection{Validation of the Hysteretic Model}

By employing the optimization routine developed in Section 5, the hysteretic model parameters $\left\{c, k_{p}\right\}$ are determined and listed in Table 3. Figure 7 compares the hysteretic model against the measured data. As illustrated, the hysteretic model is in close agreement with the data.

Table 3: Optimized parameters for the hysteretic model.

\begin{tabular}{|c|cc|}
\hline Parameter & $c$ & $k_{p}(\mathrm{~J})$ \\
\hline Optimized value & 0.2 & 430.0 \\
\hline
\end{tabular}

The performance of the hysteretic model hinges on two sources. First, the averaged curves underlie the hysteresis model. Thus, errors in the calculation of the averaged curves (i.e., in the nonhysteretic model) will propagate to the hysteretic response. For instance, the saturation regimes of the curves corresponding to a compressive stress of 8.00 MPa in Figures 7(b) and 4(b) appear to have the same error. This illustrates the benefit to improving the nonhysteretic model.

The second source is how to control the width of the hysteresis, which, as per Evans and Dapino [11], is done through the employment of the parameters $c$ and $k_{p}$. Ideally, to get the hysteretic curves from the proposed nonhysteretic model, the hysteretic model must apply a symmetric shift about the averaged curve obtained from the nonhysteretic model. However, there is no guarantee that the current hysteretic model does so. For example, consider the curve corresponding to a bias field of $1.85 \mathrm{kA} / \mathrm{m}$ in Figure 4(c), for which the nonhysteretic model accurately predicts the lower saturation magnetization. In this region, the hysteretic model should have a negligible effect, as demonstrated in Figure 7(c). However, the hysteretic model shifts the response away from the accurately calculated averaged curve. In addition, the hysteretic model overestimates the hysteresis width for sensing cases $(7(\mathrm{c}, \mathrm{d}))$, but underestimates it for actuation cases $(7(\mathrm{a}, \mathrm{b}))$. These problems suggest that the parameters $c$ and $k_{p}$ control more than just the hysteresis width. The development of a hysteresis model that overcomes these issues is left for future research. 


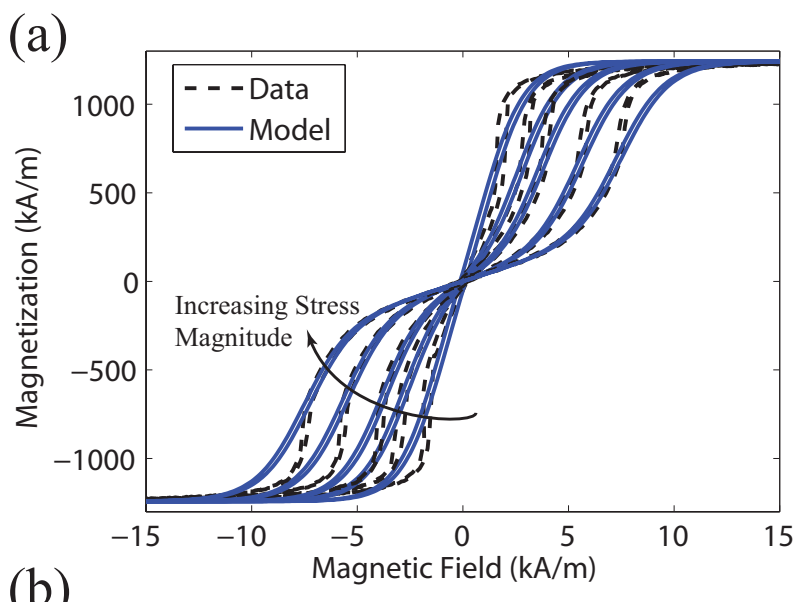

(b)

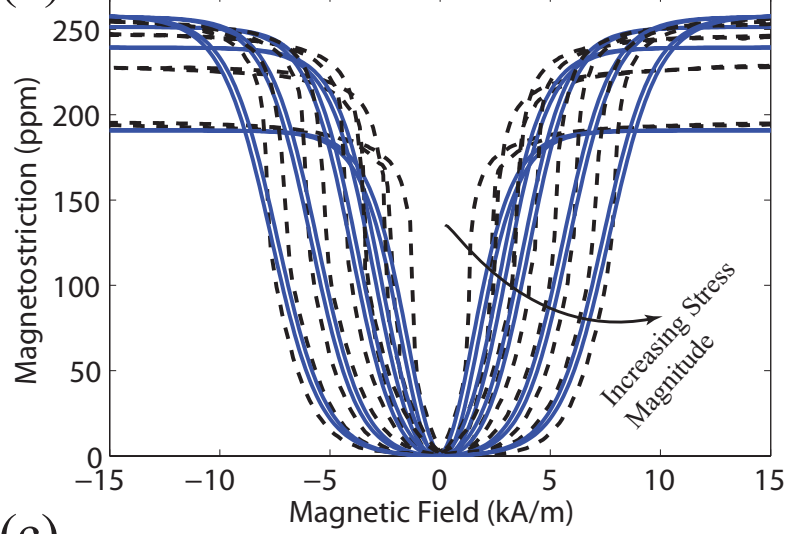

(c)

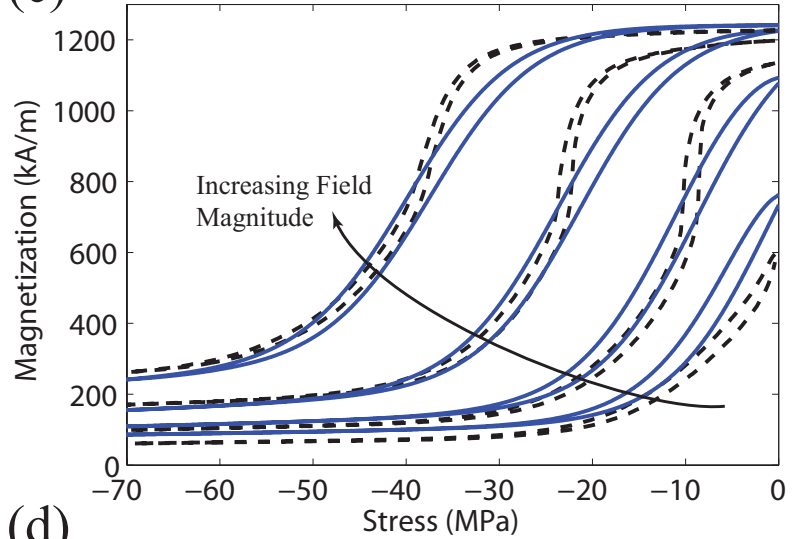

(d)

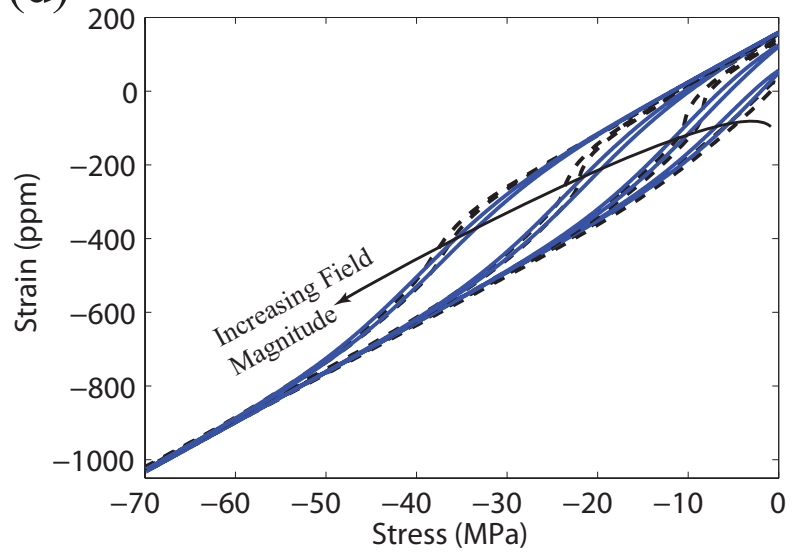

Figure 7: Comparison of hysteretic measurements and hysteretic model for $\langle 100\rangle \mathrm{Fe}_{81.5} \mathrm{Ga}_{18.5}$ grown with FSZM at (a),(b) constant compressive stress values of $0.32,8.0 .2213 .40,23.10$, and $32.30 \mathrm{MPa}$, and (c),(d) constant field values of $1.85,3.24,5.65$, and $8.88 \mathrm{kA} / \mathrm{m}$. Strain is the superposition of the mechanical strain $(\mathrm{T} / \mathrm{E})$ and the magnetostriction $(\lambda)$. 


\section{Conclusions}

Several improvements were proposed for the Discrete Energy-Averaged model, which calculates the 3D hysteretic magnetization and magnetostriction of iron-gallium alloys. In particular, the magnetoelastic energy was enhanced through the utilization of the more thorough expression of the magnetostriction tensor, and the Gibbs free energy was simplified through the reduction of the magnetic stiffness matrix. An analytical solution procedure based on an eigenvalue decomposition was then presented. It was shown that the exact solution procedure offers multiple (up to the number of easy directions) local minimum energy directions for each individual easy direction. These additional minima provide two advantages. First, the singularities that are present in the existing approximate solution are avoided. Second, the material's complex response to arbitrary stress and magnetic field inputs can be better understood.

The resulting robustness comes at an expense. For general loadings, the model requires the solution of six $6^{\text {th }}$ order polynomials. As a result, the exact solution procedure is about two times slower than the approximate procedure for 3D applications. However, analytical reductions of the model were presented for 2D and 1D applications. In the simplest case (i.e., 1D applications), only a single 4th order polynomial must be solved, thus making the exact solution procedure as efficient as the approximate procedure.

Another novelty is in finding the model parameters. Rather than employing anhysteretic curves, our approach utilizes the average of the hysteretic data, which obviates taking additional measurements. A novel optimization routine was developed that retains the number of model constants, but decouples the parameters into exclusive sets. Consequently, the set $\left\{M_{s}, E, \lambda_{100}\right\}$ was found efficiently and accurately through a preprocessing step, which fits the magnetization and magnetostriction saturation regimes with lines. Only the remaining parameters were estimated through a global optimization procedure that used a sophisticated objective error function. The effectiveness of the model was verified through comparison with existing measurement data. The hysteretic model has two sources of error: the nonhysteretic model and the hysteresis formulation itself. Even though the nonhysteretic model closely agreed with the averaged hysteretic data, the observed error propagated to the hysteretic simulations. Additionally, the parameters of the hysteretic model, which were previously thought to control only the hysteresis width, appeared to introduce unexpected error. For instance, the hysteresis model added error to the nonhysteretic magnetization response in the lower saturation region, where the hysteresis effects should have been negligible. Further research is required to develop a hysteresis model that avoids these issues. 


\section{Acknowledgments}

The authors wish to acknowledge the NASA Aeronautics Scholarship Program; a DOE GATE fellowship from the U.S. DOE GATE Center of Excellence, DE FG2605NT42616, at The Ohio State University; and the member organizations of the Smart Vehicle Concepts Center (www.SmartVehicleCenter.org), a National Science Foundation Industry/University Cooperative Research Center (I/UCRC).

\section{References}

[1] A. E. Clark, M. Wun-Fogle, J. B. Restorff, T. A. Lograsso, Magnetic and magnetostrictive properties of Galfenol alloys under large compressive stresses, in: PRICM-4: Int.Symp. on Smart Materials-Fundamentals and system Applications, Pacific Rim Conf. on Advanced Materials and Processing, 2001.

[2] J. Atulasimha, A. B. Flatau, A review of magnetostrictive iron-gallium alloys, Smart Materials and Structures 20 (4) (2011) 043001.

[3] T. V. Jayaraman, N. Srisukhumbowornchai, S. Guruswamy, M. L. Free, Corrosion studies of single crystals of iron-gallium alloys in aqueous environments, Corrosion Science 49 (10) (2007) 4015-4027.

[4] F. Preisach, Über die magnetische Nachwirkung, Zeitschrift für Physik 94 (5-6) (1935) 277302.

[5] A. Globus, Universal hysteresis loop for soft ferrimagnetic material, Proc. Europ. Physical Society, Conference on Soft Magnetic Material 2 (1975) 233.

[6] D. C. Jiles, D. L. Atherton, Theory of ferromagnetic hysteresis, Journal of Magnetism and Magnetic Materials 61 (1986) 48-60.

[7] E. C. Stoner, E. P. Wohlfarth, A mechanism of magnetic hysteresis in heterogeneous alloys, IEEE Transactions on Magnetics 27 (4) (1991) 3475-3518.

[8] F. Liorzou, B. Phelps, D. L. Atherton, Macroscopic models of magnetization, IEEE Transactions on Magnetics 36 (2) (2000) 418-428.

[9] W. D. Armstrong, An incremental theory of magneto-elastic hysteresis in pseudo-cubic ferromagnetostrictive alloys, Journal of Magnetism and Magnetic Materials 263 (2003) 208-218. 
[10] J. Atulasimha, G. Akhras, A. B. Flatau, Comprehensive three dimensional hysteretic magnetomechanical model and its validation with experimental $\langle 110\rangle$ single-crystal iron-gallium behavior, Journal of Applied Physics 103 (7) (2008) 07B336.

[11] P. G. Evans, M. J. Dapino, Efficient magnetic hysteresis model for field and stress application in magnetostrictive Galfenol, Journal of Applied Physics 107 (6) (2010) 063906.

[12] P. G. Evans, M. J. Dapino, Measurement and modeling of magnetic hysteresis under field and stress application in irongallium alloys, Journal of Magnetism and Magnetic Materials 330 (2013) 37-48.

[13] S. Chakrabarti, Modeling of 3D magnetostrictive systems with application to Galfenol and Terfenol-D transducers, Ph.D. Dissertation, The Ohio State University, 2011.

[14] S. Chakrabarti, M. J. Dapino, Nonlinear finite element model for 3D Galfenol systems, Smart Materials and Structures 20 (10) (2011) 105034.

[15] G. Engdahl, Handbook of giant magnetostrictive materials, Academic Press, San Diego, CA, 2000 .

[16] C. Kittel, Physical Theory of Ferromagnetic Domains, Rev. Mod. Phys. 21 (1949) 541-583.

[17] P. G. Evans, M. J. Dapino, Dynamic model for 3-D magnetostrictive transducers, IEEE Transactions on Magnetics 47 (1) (2011) 221-230.

[18] T. H. Cormen, C. E. Leiserson, R. L. Rivest, C. Stein, Introduction to algorithms, MIT Press, Cambridge, Mass., 2009.

[19] P. Bratley, B. Fox, Algorithm 659: Implementing Sobol's quasirandom sequence generator, ACM Transactions on Mathematical Software 14 (1) (1988) 88-100.

[20] A. Saltelli, P. Annoni, I. Azzini, F. Campolongo, M. Ratto, S. Tarantola, Variance based sensitivity analysis of model output. Design and estimator for the total sensitivity index, Computer Physics Communications 181 (2) (2010) 259-270. 


\section{A. Gibbs Free Energy Simplification}

As a result of the exact solution procedure, the Gibbs free energy (eq. (1)) can be simplified using the unity norm constraint on the magnetization directions (eq. (8b)),

$$
\begin{aligned}
\stackrel{k}{G} & =\frac{1}{2} \stackrel{k}{\mathbf{m}} \cdot \overline{\mathbf{K}} \stackrel{k}{\mathbf{m}}-\stackrel{k}{\mathbf{b}} \cdot \stackrel{k}{\mathbf{m}}+\frac{1}{2} K+\stackrel{k}{K_{0}} \\
& =\frac{1}{2} \stackrel{k}{\mathbf{m}} \cdot \mathbf{K} \stackrel{k}{\mathbf{m}}-\stackrel{k}{\mathbf{b}} \cdot \stackrel{k}{\mathbf{m}}+K+\stackrel{k}{K}_{0}
\end{aligned}
$$

where $\mathbf{K}$ is given in eq. (19). Since the volume fractions (eq. (14)) are invariant when the same constant is added to each energy $\stackrel{k}{G}$, eq. (31) can be simplified as,

$$
\stackrel{k}{G}=\frac{1}{2} \stackrel{k}{\mathbf{m}} \cdot \mathbf{K} \stackrel{k}{\mathbf{m}}-\stackrel{k}{\mathbf{b}} \cdot \stackrel{k}{\mathbf{m}}+\stackrel{k}{K_{0}},
$$

without loss of generality. eq. (32) can be further simplified by taking the dot product of eq. (20a) with $\stackrel{k}{\mathbf{m}}$, solving for the quadratic term, and using eq. (20b) to write,

$$
\stackrel{k}{\mathbf{m}} \cdot \mathbf{K} \stackrel{k}{\mathbf{m}}=\stackrel{k}{\gamma}+\stackrel{k}{\mathbf{b}} \cdot \stackrel{k}{\mathbf{m}}
$$

Substituting eq. (33) into eq. (32) gives,

$$
\stackrel{k}{G}=\frac{1}{2}(\stackrel{k}{\gamma}-\stackrel{k}{\mathbf{b}} \cdot \stackrel{k}{\mathbf{m}})+\stackrel{k}{K_{0}} .
$$

